\title{
Electroactive Nanoporous Metal Oxides and Chalcogenides by Chemical Design
}

\author{
Christopher H. Hendon, ${ }^{\dagger, \ddagger}$ Keith T. Butler, ${ }^{\ddagger}$ Alex M. Ganose, ${ }^{\S}, \|$ Yuriy Román-Leshkov, ${ }^{\dagger}$
} David O. Scanlon, ${ }^{\S, \| \odot ~ G e o f f r e y ~ A . ~ O z i n, ~}{ }^{\perp}$ and Aron Walsh*, ${ }^{*, \nabla_{\odot}}$

${ }^{\dagger}$ Department of Chemical Engineering, Massachusetts Institute of Technology, Cambridge, Massachusetts 02139, United States

${ }^{\ddagger}$ Department of Chemistry, University of Bath, Claverton Down, Bath, BA2 7AY, United Kingdom

${ }^{\S}$ Kathleen Lonsdale Materials Chemistry, Department of Chemistry, University College London, 20 Gordon Street, London, WC1H 0AJ, United Kingdom

"Diamond Light Source Ltd., Diamond House, Harwell Science and Innovation Campus, Didcot, Oxfordshire OX11 0DE, United Kingdom

${ }^{\perp}$ Department of Chemistry, University of Toronto, Toronto, Ontario M5S 3H6, Canada

\#Department of Materials Science and Engineering, Yonsei University, Seoul 03722, South Korea

${ }^{\nabla}$ Department of Materials, Imperial College London, Exhibition Road, London SW7 2AZ, United Kingdom

\section{Supporting Information}

\begin{abstract}
The archetypal silica- and aluminosilicate-based zeolite-type materials are renowned for wide-ranging applications in heterogeneous catalysis, gas-separation and ion-exchange. Their compositional space can be expanded to include nanoporous metal chalcogenides, exemplified by germanium and tin sulfides and selenides. By comparison with the properties of bulk metal dichalcogenides and their 2D derivatives, these open-framework analogues may be viewed as three-dimensional semiconductors filled with nanometer voids. Applications exist in a range of molecule size and shape discriminating devices. However, what is the electronic structure of nanoporous metal chalcogenides? Herein, materials modeling is used to describe the properties of a homologous series of nanoporous metal chalcogenides denoted

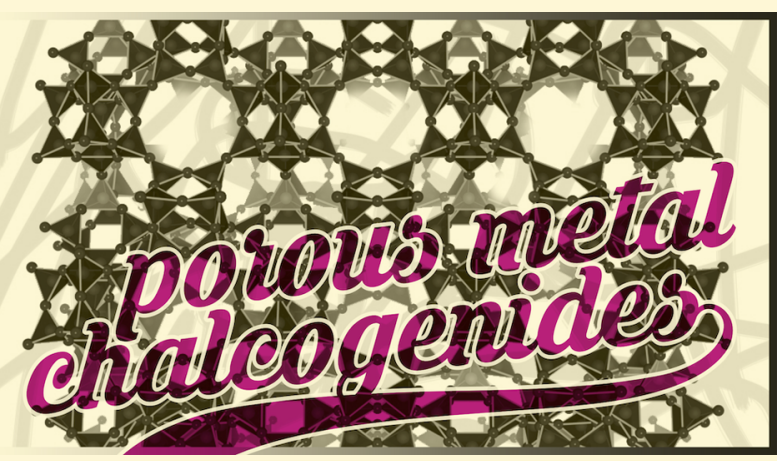
$\mathrm{np}-\mathrm{MX}_{2}$, where $\mathrm{M}=\mathrm{Si}, \mathrm{Ge}, \mathrm{Sn}, \mathrm{Pb}$, and $\mathrm{X}=\mathrm{O}, \mathrm{S}, \mathrm{Se}$, Te, with Sodalite, LTA and aluminum chromium phosphate- 1 structure types. Depending on the choice of metal and anion their properties can be tuned from insulators to semiconductors to metals with additional modification achieved through doping, solid solutions, and inclusion (with fullerene, quantum dots, and hole transport materials). These systems form the basis of a new branch of semiconductor nanochemistry in three dimensions.
\end{abstract}

\section{INTRODUCTION}

The concept of reconstructing a bulk semiconductor through chemical synthesis into a semiconductor containing a periodic array of nanopores was introduced in $1992 .{ }^{1}$ The vision was a new genre of semiconductor device with electronic, optoelectronic, and optical properties that were sensitive to the size and shape of molecules adsorbed within the nanopores. The archetypes of this class of zeolitic semiconductors (ZSs) were based on germanium and tin sulfide and selenide compositions. They were found to have open-framework structures, reminiscent of silica- and alumina-based zeolites, comprising tetrahedrally connected networks of tetrahedral metal chalcogenide building blocks. ${ }^{2}$ Inspired by this knowledge, their potential as electronic sensors was explored; ${ }^{3}$ however, information about their electronic structure, which is key to establishing their physical structure, property, function, and utility relations, remains largely unknown today.
The synthesis of ZSs is as challenging as it is important. In the first decade of this century, great efforts were expended to produce alternative zeolitic materials by replacing oxygen with chalcogens and substituting the ubiquitous $\mathrm{Si}$ and $\mathrm{Al}$ with alternate tetrahedral metal centers. ${ }^{4-6}$ Further structural diversity-including the isolation of metastable and unstable structures-was realized by application of structure directing agents thereby expanding the structural library. ${ }^{7}$ Structurally, the incorporation of germanium allowed a proliferation of new frameworks and the synthesis of ultraporous framework materials. ${ }^{8,9}$ Moreover, the highly polarizable nature of chalcogen atoms was found to result in enhanced gas absorption in these systems. ${ }^{10,11}$ Latterly, alternative synthetic

Received: February 6, 2017

Revised: $\quad$ March 27, 2017

Published: March 27, 2017 
routes, such as ionothermal synthesis and post-synthesis reduction, have reinvigorated the search for new framework structures and compositions. ${ }^{12-15}$ The recent report of measured semiconducting behavior in a zeolite-analogue material, ${ }^{16}$ as well as more exotic electronic phenomena such as anomalous band gap evolution in nanocrystalline topological insulators, ${ }^{17}$ shows that this field offers great potential. One very recent study demonstrated the synthesis of open-frame ZSM-5 high tin sulfide and selenide zeolite analogues, ${ }^{18}$ with surface areas and stabilities equivalent or superior to zeolites. The confluence of novel synthetic methods, early reports of semiconducting behavior, and advances in modern computational techniques have inspired us to address the electronic structure of these materials.

We report the electronic properties of nanoporous metal chalcogenides ( $n p-\mathrm{MX}_{2}$, where $\mathrm{M}=\mathrm{Si}, \mathrm{Ge}, \mathrm{Sn}, \mathrm{Pb} ; \mathrm{X}=\mathrm{O}, \mathrm{S}$, $\mathrm{Se}, \mathrm{Te}$ ) exemplified by the sodalite (SOD), Linde Type A (LTA) and aluminum chromium phosphate-1 (ACO) structures and compare these properties with those of the bulk forms $\left(\mathrm{MX}_{2}\right)$. The electronic structure is calculated from first-principles within Density Functional Theory (DFT), a predictive tool in contemporary materials design. ${ }^{19,20} \mathrm{We}$ assess the nanoporous and bulk forms of the simplest metal chalcogenides, the corresponding metal oxides (np- $\mathrm{MO}_{2}$ and $\mathrm{MO}_{2}$ ) and mixed anion and cation solid solutions. The results allow insights into how the structure and composition of nanoporous metal chalcogenides determine their electrical and optical properties, and provide a robust platform for developing "inverse quantum dot" nanoporous semiconductors.

\section{STRUCTURAL STABILITY}

Before we consider the electronic structure, it is pertinent to assess the stability of the phases. We emphasize that the formation of most porous solids is kinetically controlled, with nucleation and templating being key in the crystallization process. Nonetheless, the thermodynamic stability relative to known siliceous zeolites is an important comparison, which has been made before to assess the viability of hypothetic framework structures. ${ }^{21}$

First of all, all structures were found to be dynamically stable with no negativity frequency phonon modes at the center of the Brillouin zone. To address thermodynamic stability, we have calculated the total energies of the three framework types and compare them to the corresponding bulk crystal ground-state structures. The data is presented in Table S1. The pure silica forms of ACO, SOD, and LTA are known to exist ${ }^{22,23}$ and can be accessed through hydrothermal synthesis routes, employing structure directing agents (SDAs or templates) as required. We characterize the stability of the frameworks relative to the ground-state crystal structure of the same stoichiometry by calculating the energy difference per atom. For the ACO, SOD, and LTA frameworks of $\mathrm{SiO}_{2}$ the enthalpic cost associated with the formation of the porous solid is $0.55,0.54$, and $0.55 \mathrm{eV}$ per formula unit, respectively.

The $\mathrm{SiO}_{2}$-ZMS-5 material was also included in our energetic assessment, as an industrially relevant material, and was found to be highly stable with a formation enthalpy cost of $0.004 \mathrm{eV}$ per formula unit relative to $\mathrm{SOD}-\mathrm{SiO}_{2}$. This stabilization is attributed to the reduction in acute $\mathrm{M}-\mathrm{O}-\mathrm{M}$ bonding angles. These angles are critical descriptors in the assessment of stability of larger metal and chalcogenide-containing structures, where more larger metal/chalcogenides favor more obtuse MO-M angles. ${ }^{24-26}$ ZSM-5 was not studied in all compositional arrangements because its large unit cell presented computational challenges for the heavier metal-chalcogenides. However, the electronic properties of the $\mathrm{ZSM}-5-\mathrm{SiO}_{2}$ were similar to that of ACO, SOD, and LTA. The electronic properties of the np$\mathrm{MX}_{2}$ materials are essentially unperturbed by structure-type; our computations on the smaller cell zeolites represent an upper limit in formation enthalpy.

The $\mathrm{GeO}_{2}$ frameworks display a similar stability with respect to the reference state than the $\mathrm{SiO}_{2}$ analogues with an enthalpic cost of $0.85 \mathrm{eV}$ (SOD), $0.79 \mathrm{eV}$ (LDA), and $0.84 \mathrm{eV}$ (ACO), respectively. The ability of germanium to form the same mineral structures as silicon can be understood from their similar ionic radii, electronegativities, and valence shell characteristics. $^{27}$ Indeed, large numbers of germanates that are isostructural to natural silicates have been synthesized. Some differences exist between the $\mathrm{Si}-\mathrm{O}-\mathrm{Si}$ motif and the $\mathrm{Ge}-\mathrm{O}-\mathrm{Ge}$ motif, ${ }^{25}$ for example, the $\mathrm{Ge}-\mathrm{O}$ bond is longer and the germanate angle is smaller than the silicate species. ${ }^{28}$ The difference in bond length is related to ionic radius, while the variation in bond angle can be understood in the context of the valence shell electron pair repulsion (VSEPR) model. ${ }^{29}$ In line with VSEPR, there is a greater repulsion between nonbonded electrons than bonded, thus the two nonbonded pairs force a tighter angle in the bonding electrons in the germanate structure.

There is an increase in formation enthalpy for the nanoporous sulfides and selenides. As has been noted previously, the tilt angle joining the tetrahedral building blocks of zeolite frameworks is largely fixed at $109^{\circ}$ in sulfide species, ${ }^{30}$ meaning that the formation of these frameworks involves additional strain, as reflected in the energies of $\mathrm{Si}, \mathrm{Sn}$, and $\mathrm{Ge}$ sulfide and selenide species. The $\mathrm{Pb}$ chalcogenides and $\mathrm{SiTe}_{2}$ represent a deviation from this trend. In these cases, the increased bond-lengths and higher atomic polarizability may be responsible for an enhanced flexibility. For reference, the formation enthalpies for all materials examined here are comparable to the formation enthalpy of other metastable materials (e.g., MOF-5). ${ }^{31}$

It should be further noted that chalcogenide zeolitic frameworks are known, which consist of assemblages of nanoparticle building blocks of $\mathrm{MX}_{2}$ stoichiometry. ${ }^{32-35}$ This is further suggested from our calculations; the one exception is the tellurides of which there are no reports in the solid-state with a stoichiometry of $\mathrm{MTe}_{2}$. Given the predicted accessibility of many of these species-and other more strained ring frameworks ${ }^{36}$ - the existence of a plethora of experimentally realized analogues and the need for electronic insights to assist in the realization of their true potential, we now undertake a systematic exploration of the effects of chemical and structural modifications on the band-structure of these materials.

\section{ELECTRONIC STRUCTURE}

The electronic properties of many porous materials feature localization of the electronic density of states in real space and hence a lack of dispersion in the bands in reciprocal space. This is certainly true for other porous materials (e.g., metal-organic frameworks) in which many recent publications have shown flat electronic bands in reciprocal space ${ }^{37-39}$ that can be modulated through chemical substitutions and inclusions. ${ }^{40,41}$ In the case of the porous metal oxides and chalcogenides presented here, the valence band is nondisperse (this is described by a large effective mass for hole transport: $m_{\mathrm{h}}^{*}>1 \mathrm{~m}_{\mathrm{e}}$ for $\mathrm{np}-\mathrm{SiO}_{2}$ ), composed entirely of oxygen/chalcogen p-orbitals, independent 

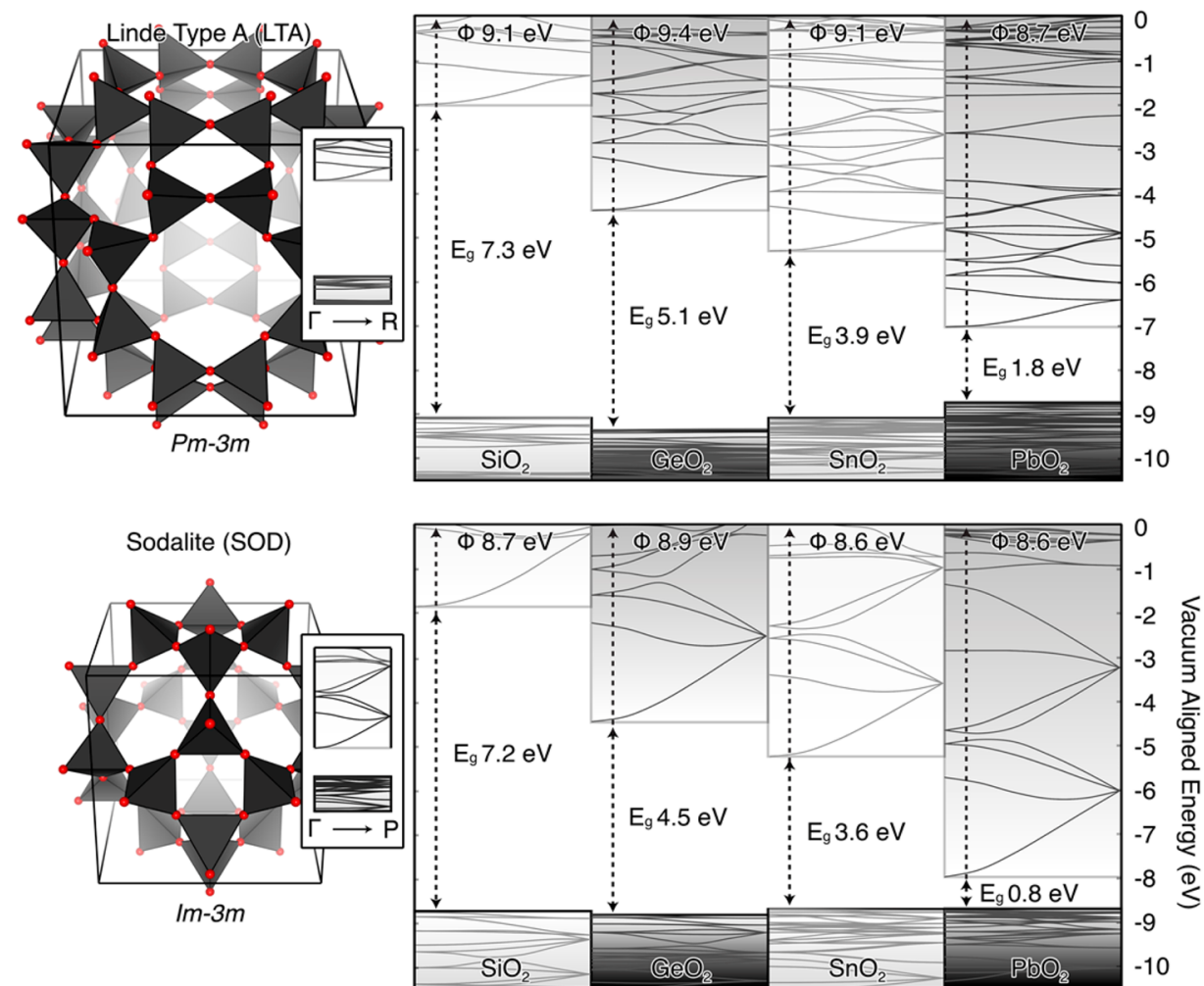

ACP-1 (ACO)
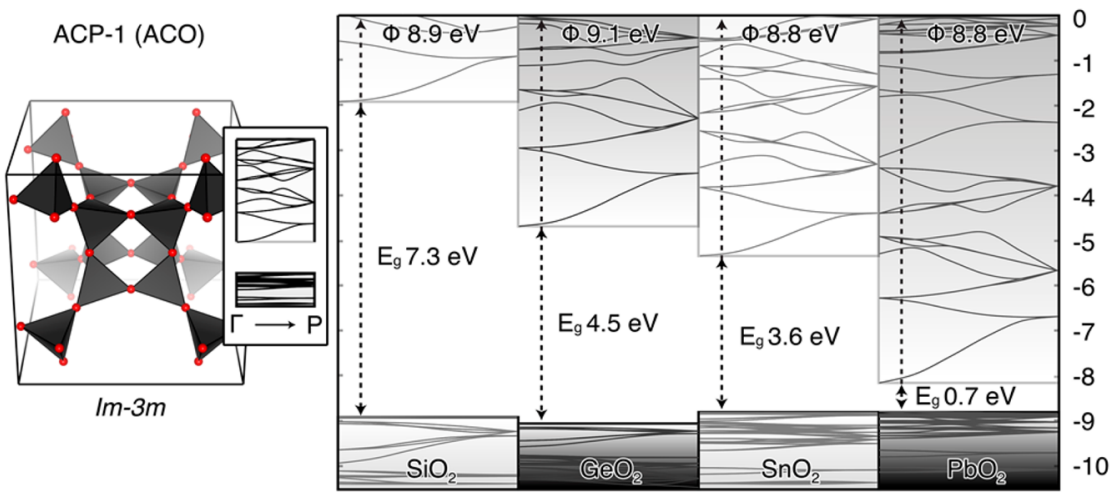

Figure 1. Electronic band structures (DFT/HSE06), aligned to the vacuum level by the ionization potential $(\Phi)$, of $\mathrm{MO}_{2}(\mathrm{M}=\mathrm{Si}, \mathrm{Ge}, \mathrm{Sn}, \mathrm{Pb})$ in the zeolite-type structures shown on the left. Plotted following $0,0,0$ to $1 / 2,1 / 2,1 / 2$ in reciprocal space (where $0,0,0=$ gamma and $1 / 2,1 / 2,1 / 2=$ special point as described in the figure), SOD structured materials show the most significant dispersion in the conduction band (ca. $2 \mathrm{eV}$ ), followed by ACO (ca. $1.6 \mathrm{eV}$ ), and LTA (ca. $0.7 \mathrm{eV}$ ). The conduction band is composed of delocalized antibonding $\mathrm{O} s$ and $\mathrm{M}$ s-orbitals. The valence band is composed of localized $\mathrm{O}$ p-orbitals, resulting in flat bands, and the bulk potential is relatively insensitive to the cation identity.

of crystal morphology. These p-orbitals are orientated into the pores and thus do not form a continuous pathway in the framework of the structure for charge transport; similar obstacles to hole mobility are often encountered in amorphous oxides. $^{42}$

Owing to the stoichiometry and IV oxidation state of the group 14 metals $\mathrm{Si}, \mathrm{Ge}, \mathrm{Sn}$, and $\mathrm{Pb}$, the lowest unoccupied states are a hybrid of unoccupied oxide/chalcogen and metal sorbitals. The crystal orbitals are delocalized throughout the structure. As a result, $\mathrm{np}-\mathrm{MX}_{2}$ materials demonstrate pronounced band dispersion (a parabolic effective mass of $m_{\mathrm{e}}^{*}=0.64 \mathrm{~m}_{\mathrm{e}}$ for $\mathrm{np}-\mathrm{SiO}_{2}$ in the SOD structure) comparable to other wide band gap semiconductors. ${ }^{43}$

The valence and conduction band edges are defined by the chalcogen and metal/chalcogen identity, respectively (Supporting Information, Figure S1). These bands can be aligned to the universal vacuum level: ${ }^{44}$ Figure 1 illustrates that the work functions of np- $\mathrm{MO}_{2}$ semiconductors are relatively independent of metal and morphology. This results from the similarities between the oxygen crystal environment-at the vertices of tetrahedra of $M(I V)$ cations-and reflects the fact that the electron energies at these sites are dominated by the Madelung potential. ${ }^{45,46}$ The electron affinity (EA) is related to the energy of the unoccupied metal center s-orbitals. The EA displays a clear trend to increase as we move to larger metals, a trend related to the relativistic effects of the nucleus on the electrons that scales with $Z^{2}$. This observed increase in electron affinity may be attributed to a contraction in the $s$ and $p$ and expansion of the $d$ and $f$ orbitals of the metal, resulting in stabilization of the low lying unoccupied states.

To isolate the effect of the anion, the electronic structure of the $\mathrm{SnX}_{2}$ series was explored, Figure 2. The ionization potential $(\Phi)$ decreases with increase in anion size but only the oxide is predicted to have a band gap. Heavier $\mathrm{np}-\mathrm{MX}_{2}$ compounds 


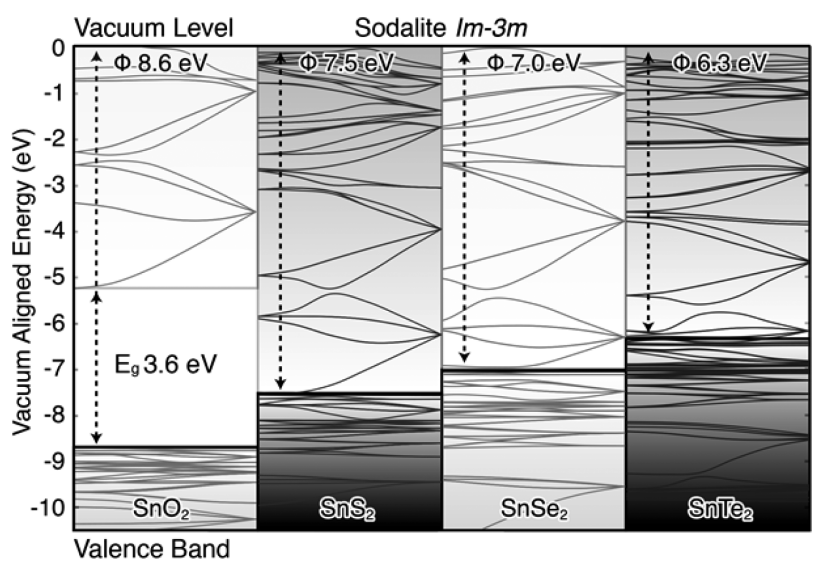

Figure 2. Electronic band structures (plotted from 0,0,0 to $1 / 2,1 / 2,1$ / 2 in reciprocal space) for sodalite $\mathrm{SnX}_{2}(\mathrm{X}=\mathrm{O}, \mathrm{S}, \mathrm{Se}, \mathrm{Te})$ relative to the vacuum level. The oxide has a band gap, while the sulfide, selenide, and telluride are metallic and their work functions are defined by their Fermi levels (in the limit of $\mathrm{T} \rightarrow 0 \mathrm{~K}$ ).

result in valence and conduction band crossing, thus forming a semimetal (a zero gap material) with a low electronic density of states at the Fermi level. This is true of all np-MX $\mathrm{MX}_{2}$ materials, with the exception of SOD-SiS 2 that has a predicted band gap of $0.2 \mathrm{eV}$. Further details of band alignments for $\mathrm{np}-\mathrm{MX}_{2}$ are provided in Table S1.

Previous studies on nanostructured $\mathrm{ZnO}$ showed a large effect of the porosity of the material on the band gap. ${ }^{47}$ Although the magnitude of the band gap of IV-VI np-MX materials is not as clearly modulated with morphology, we observe a similar tendency toward a wider band gap with increased porosity, with LTA displaying the largest gap in all cases. Moreover, conduction band dispersion is affected by nanoporosity, offering a route toward the engineering of electron transport properties. For instance, the conduction band effective mass $m_{\mathrm{e}}^{*}=0.74 \mathrm{~m}_{\mathrm{e}}$ for LTA-SiO 2 compared to $m_{\mathrm{e}}^{*}=0.64 \mathrm{~m}_{\mathrm{e}}$ for SOD-SiO 2 .

Band engineering of solid-state semiconductors by creating solid solutions (compositional alloys) is a well-established practice. To consider the effects of forming ZS alloys we treat two possible isovalent substitution routes for the SOD $\mathrm{SiO}_{2}$ framework: (i) a single extrinsic inclusion of another metal at the Si site; (ii) a single extrinsic chalcogenide substitution at an oxygen site. The results are summarized in Figure 3 (higher chalcogenide concentrations and energetics are presented in the Figure S4 and the Supporting Information, respectively). In both the metal and the anion substitution cases, the band gaps are intermediate between the binary end-points, that is, following Vegard's rule. ${ }^{48}$

The different substitution types offer precision control over which electronic property is tuned. The ionization potential can be altered by anion substitution, electron affinity by metal mixing, and the band gap by either or both. In all cases the band engineering is achieved by introducing well-defined states in the gap of the host material. Doping on the chalcogen site leaves the intrinsic band structure essentially unaltered and the resultant midgap state is highly localized, as evidenced by the lack of dispersion in the upper valence band (Figure 3). Note that the Te-substituted chalcogen resulted in a framework with a stress beyond the ultimate tensile strength of the framework and could not be mechanically stabilized, although solid
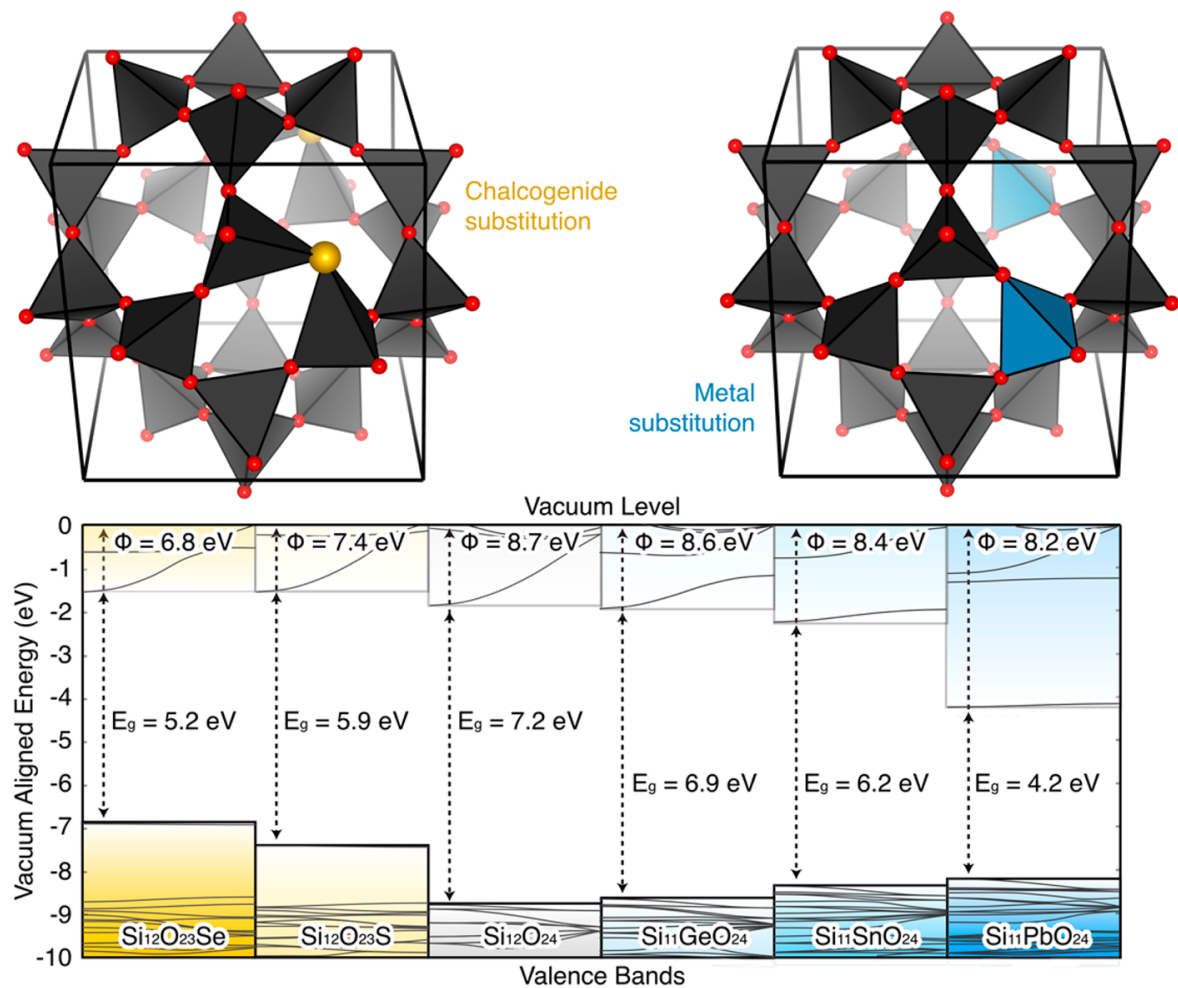

Figure 3. Isovalent dopants introduce localized states in the electronic band structure of porous metal chalcogenides (DFT/HSE06), allowing for modular electronic band gaps and ionization potentials $(\Phi)$. For example, in $\mathrm{SiO}_{2-x} \mathrm{Se}_{x}$ (left-hand side), the low binding energy of the filled Se 4p orbitals introduces a state $2 \mathrm{eV}$ above the valence of $\mathrm{SiO}_{2}$, while in $\mathrm{Si}_{1-x} \mathrm{~Pb}_{x} \mathrm{O}_{2}$ (right-hand side), the high binding energy of the unoccupied $\mathrm{Pb} 6 \mathrm{~s}$ orbital introduces a state $3 \mathrm{eV}$ below the conduction band of $\mathrm{SiO}_{2}$. 
solutions with heavier chalcogen frameworks (e.g., $\mathrm{MSe}_{2-x} \mathrm{Te}_{x}$ ) may be possible.

The metal substitution induced gap state shows a trend toward increased localization as we move further in chemical space from the original host species. The dispersion for the Ge defined conduction band is similar to that in purely siliceous SOD. The band dispersion in reciprocal decreases as we move down the column of the periodic table, until the flat, midgap state of $\mathrm{Pb}$. The trend can be understood in terms of atomic orbital mixing and overlap in forming the crystal wave functions. As the metals become more chemically distinct the degree of mixing is reduced and the state defining the band edge participates to a lesser extent in the interconnected matrix of the framework. This observation has implications for charge transport in the material, as higher effective masses will reduce electron mobility and hence conductivity.

\section{SEMICONDUCTOR APPLICATIONS}

Thus far we have considered the properties of ZSs with respect to conventional solid-state semiconductors. However, the trends and properties that have been revealed are of interest in their own right, provoking the question: what is the nanoporous semiconductor advantage?

The answer lies in the void, with the availability of a continuous, ordered, porous, conducting network. These systems can be intimately mixed with materials possessing complementary electronic properties. This concept opens up a plethora of combinations and opportunities. To illustrate the potential for forming composite systems we initially consider a prototypical pore-filling material: fullerene, ubiquitous in organic electronics. In combination with ZSs, we demonstrate how it can find enhanced application. Figure 4 shows the upper valence electron density of $\mathrm{LTA}-\mathrm{SiO}_{2}$ with a fullerene in the pore, ${ }^{49}$ along with the associated electron energies, on the lefthand side the energy levels. The straddling type-I offset of the isolated materials is reproduced in the composite system. This type of junction is widely applied in quantum dots and wells to stabilize excitons and provide enhanced luminesce in the confined system. The arrangement means that both holes and electrons are blocked from leaving the fullerene, yet the fullerenes can still form an ordered array, afforded by the framework structure of the ZS. We note that during the preparation of this manuscript an exciting report of $\mathrm{Ag}$ nanoparticles contained within zeolitic- $\mathrm{SiO}_{2}$ was reported and demonstrated unusual quantum confinement effects, ${ }^{50}$ substantiating this potential application.

Type-II offsets or staggered band gaps alignment is perhaps the most desirable alignment regime for semiconductor devices, finding application for example in bipolar transistors, light emitting diodes, and photovoltaic devices. This type of offset allows for robust separation of charges (electrons and holes) between the two materials. To illustrate the possibility of utility of ZSs with other nanotechnologies we demonstrate the alignment of $\mathrm{SOD}-\mathrm{SnO}_{2}$ with $\mathrm{PbS}$ quantum dots in Figure $4 \mathrm{~b}$. In this case the size of the quantum dot as well as the porosity and composition of the framework allows for fine-tuning of the alignments. One application is the synthesis of photovoltaic absorber layers with the high interfacial areas associated with bulk heterojunction solar cells but with a degree of order and regularity unobtainable in typical donor-acceptor composites. This in turn could lead to enhanced charge separation, carrier lifetimes, and ultimately device performance. For the compositions considered here the frameworks would be best

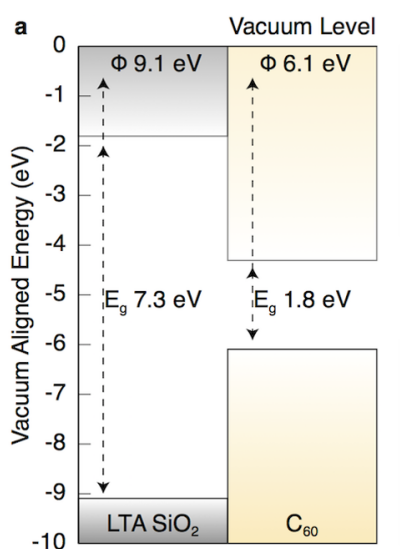

b
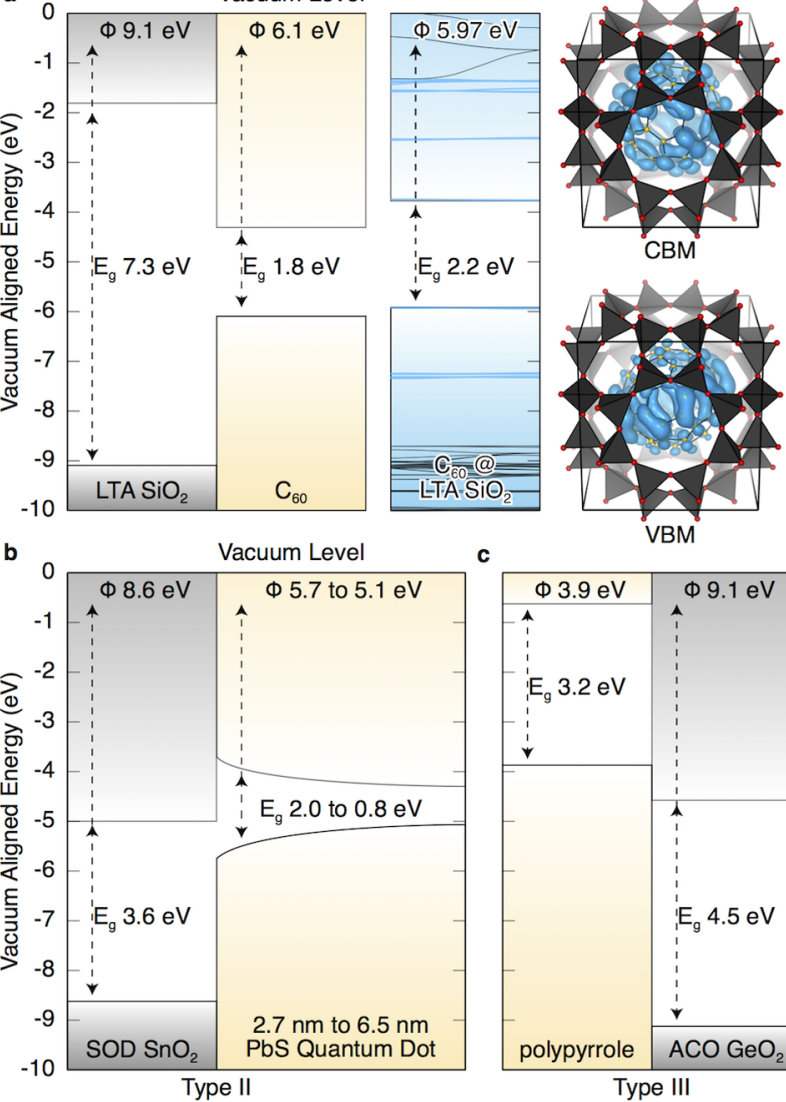

CBM

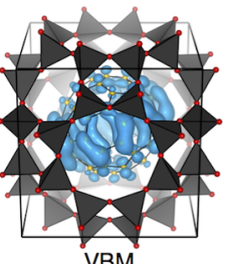

c

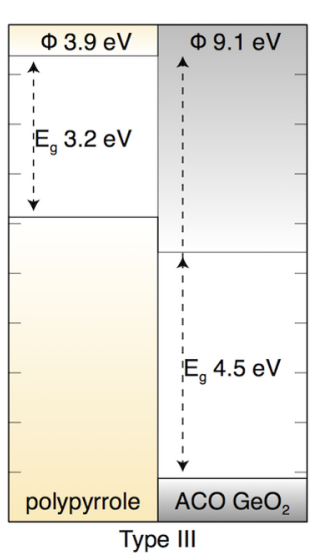

Figure 4. Representations of (a) $\mathrm{C}_{60} @ \mathrm{LTA}-\mathrm{SiO}_{2}$ forming a type I semiconductor internal heterojunction, with strong quantum confinement which could provide enhanced exciton lifetimes and luminescence. The calculated offsets based on the isolated and composite systems are shown (blue bands corresponding to $\mathrm{C}_{60^{-}}$ centered bands). SOD-SnO 2 pores $(d=10.7 \AA)$ may be filled with small $\mathrm{PbS}$ quantum dots, where confinement effects can be exploited to change the nature of the band offsets. Here we show the broadening of the electronic gap in slightly larger $\mathrm{PbS}$ quantum dots and can project that the smaller dots will form the type-II semiconductor heterojunction. Loading $\mathrm{ACO}-\mathrm{GeO}_{2}$ with the conductive polymer, polypyrrole (c), should form a type-III semiconductor heterojunction, which could lead to selective hole transport in the polypyrrole layer and electron injection into the $\mathrm{GeO}_{2}$ framework.

suited to acting as n-type semiconductors in such an architecture, because of the greater dispersion and lower effective mass in the conduction band as opposed to the valence. Design rules for p-type frameworks could include the use of metal cations with lone-pair s-electrons constituting the valence band maximum, similar principles have been explored for establishing p-type transparent conductive oxides. ${ }^{51}$

Type-III offsets usually occur in materials with contrasting electronegativities, in this case the valence band of one material lies closer to the vacuum level than the conduction band of the other material, resulting in a spontaneous transfer of charge at the interface. Such architectures have been proposed for biomedical applications; however, these offsets are uncommon in traditional semiconductors ${ }^{52,53}$ as the change in electronegativity is generally accompanied by a large difference in crystal structure. Recent developments in epitaxial growth of nanowires have opened up new possibilities for defect-free type-III interfaces. ${ }^{54,55}$ The application of ZSs with semiconducting inclusion materials offers another route to the 
fabrication of this important class of semiconductor architectures. $\mathrm{LTA}-\mathrm{SiO}_{2}$ with polypyrrole in the pore is depicted in Figure 4c. This case corresponds to a type-III offset, with the valence bands of the polypyrrole higher in energy than the conduction band of the ZS, due to the destabilizing effects of the lone pair on the nitrogen site.

Another potential application of the frameworks is as transparent conductors (TCs). Traditionally TCs have relied on taking a wide gap material (usually an oxide) and tuning the carrier levels to induce conductivity. Recently, however, Zunger and co-workers ${ }^{56}$ demonstrated a new route to achieving TCs through the control of interband transitions in metallic conductors. The proposed type 2 intrinsic conductors are semimetals with large direct bandgaps and zero indirect bandgaps. The $\mathrm{SnX}_{2}$ series presented in Figure 2 demonstrate how this kind of band structure is achievable in porous chalcogenides. Through the choice of metal and anion the interband transitions can be tuned to be symmetry allowed or forbidden, suggesting the possible application of ZSs as transparent intrinsic conductors.

The applications outlined above represent a select few examples; the field is limited only by the imagination of its practitioners. We have demonstrated the thermodynamic feasibility of electroactive porous chalcogenide frameworks, and recent work in the field of infiltrated carbon nanotubes ${ }^{57}$ and electro-activated $\mathrm{MOFs}^{58}$ already demonstrates the wide array of novel technologies made possible by such strategies. The gauntlet is now thrown down to engineers to design and develop novel applications, to physicists to explore and explain the rich electronic structure, and to chemists to synthesize and realize these configurations.

\section{CATALYTIC APPLICATIONS}

While the substitution of metallic heteroatoms into the microporous matrix of zeolites has resulted in a large number of novel catalytic systems, the isomorphic substitutions of the bridging oxygen atoms in these materials by other elements, such as $\mathrm{S}$, Se, and $\mathrm{Te}$, to produce microporous metal chalcogenides has seldom been explored and continues to be an exciting topic in the context of catalytic bond activation. ${ }^{59}$ This lack of understanding exists despite our ability to, on the one hand, synthesize well-defined metal chalcogenide structures, and on the other hand, use such materials with open architectures as catalysts in a variety of photo- and electrochemical applications. $^{60}$

An opportunity exists for the use of microporous metal chalcogenides to generate exotic catalytic environments that combine the confinement effects of microporous materials with the electronic properties generated by the metal-chalcogen motifs. Indeed, microporous environments can discriminate chemical pathways by allowing the diffusion of molecules with the correct shape and size. The pores and voids of these materials have molecular dimensions capable of stabilizing reactive intermediates and transitions states that mediate chemical reactivity by van der Waals interactions. This is reminiscent of the solvation effects prevalent within enzyme pockets and has analogous consequences for catalytic specificity. ${ }^{61}$ The topochemical characteristics of the pores mediate enthalpy-entropy compromises that determine Gibbs free energies of the reactive adducts, thus extending the diversity of the microporous solid matrix well beyond simple size discrimination allowing the utilization of entropy as a design principle. ${ }^{62}$ Microporous environments have been exploited to stabilize late transition metal clusters via encapsulation to generate bifunctional catalysts. ${ }^{63}$ Notably, the porous materials described herein can be synthesized with tunable bandgaps, making them suitable as selective photoredox catalysts. Taken together, all these properties can be combined to address a variety of challenging transformations. For example, these catalysts are promising materials for the selective removal of endocrine disruptor compounds-water-soluble organic pollutants known for their ability to mimic hormones, leading to adverse health effects on aquatic organisms ${ }^{64}$ and in the early stages of human life ${ }^{65}$ even at exceedingly low (i.e., $\mathrm{pg} / \mathrm{L}$ ) concentrations-from wastewater streams via chemoselective photodegradation in the presence of other organic species. Interestingly, the decomposition of organic waste could lead to the production of fuels and electricity when using these materials in photofuel-cells. ${ }^{66}$ Similar approaches could be implemented for the removal of air pollutants in vehicles and buildings. Further, the cooperative effects between the semiconducting framework with encapsulated redox-active clusters can be exploited for light-assisted bond activation in thermocatalysis (an example is shown in Figure S3), akin to the cooperative effect attained with localized surface plasmons to lower activation barriers and increase the selectivity by allowing the thermocatalytic production of sensitive compounds at lower temperatures. $^{67,68}$

\section{CONCLUSION}

From a predictive materials modeling investigation, we have shown that nanoporous oxides and chalcogenides exhibit an impressive diversity in their electronic behavior. Depending on the choice of metal or chalcogen, the band gaps can be tuned from the regime of wide band gap insulators to semiconductors, with metallicity observed for the heavier chalcogens. Perhaps these heavier chalcogenide materials could be interesting electrically conductive materials. These properties can be further tuned through doping and solid solutions, and their porosity can be exploited to construct a range of electronic offsets within the materials by using electroactive guests. The thermodynamic cost of forming the chalcogenide frameworks is similar to known nanoporous materials, and they should be amenable to the full range of established synthetic techniques including chemical templating. Furthermore, the diversity and chemical modularity provides unusual routes toward high surface area heterogeneous catalysts with compelling compositions for photo-, electro-, and thermal catalytic applications. Thus, this class of porous metal chalcogenides has the potential to bring semiconductor nanochemistry firmly into three dimensions.

\section{ASSOCIATED CONTENT}

\section{S Supporting Information}

The Supporting Information is available free of charge on the ACS Publications website at DOI: 10.1021/acs.chemmater.7b00464.

Further details on the computational methods and a table of the calculated electronic and structural properties of each material (PDF)

\section{AUTHOR INFORMATION}

\section{Corresponding Author}

*a.walsh@imperial.ac.uk. 


\section{ORCID}

Christopher H. Hendon: 0000-0002-7132-768X

Yuriy Román-Leshkov: 0000-0002-0025-4233

David O. Scanlon: 0000-0001-9174-8601

Aron Walsh: 0000-0001-5460-7033

Notes

The authors declare no competing financial interest.

\section{ACKNOWLEDGMENTS}

A.W., K.T.B., and C.H.H. are supported by the Royal Society, the EPSRC (Grant EP/M009580/1) and the ERC (Grant 277757). D.O.S. and A.W. acknowledge membership of the Materials Design Network. A.M.G. acknowledges Diamond Light Source for the cosponsorship of a studentship on the EPSRC Centre for Doctoral Training in Molecular Modelling and Materials Science (EP/L015862/1). GAO is a Government of Canada Research Chair in Materials Chemistry and Nanochemistry. He deeply appreciates the strong and sustained support of his research from the Natural Sciences and Engineering Research Council of Canada. This work benefitted from access to the ARCHER, through the UK High Performance Computing Consortium, which is funded by EPSRC Grant EP/L000202, and access to XSEDE Grant Number AC-1053575.

\section{REFERENCES}

(1) Ozin, G. A. Nanochemistry: Synthesis in Diminishing Dimensions. Adv. Mater. 1992, 4, 612-649.

(2) Han, C.; Sun, Q.; Li, Z.; Dou, S. X. Thermoelectric Enhancement of Different Kinds of Metal Chalcogenides. Adv. Energy Mater. 2016, 6, 1600498.

(3) Zheng, N.; Bu, X.; Wang, B.; Feng, P. Microporous and Photoluminescent Chalcogenide Zeolite Analogs. Science 2002, 298, 2366-2369.

(4) Trikalitis, P. N.; Rangan, K. K.; Bakas, T.; Kanatzidis, M. G. Varied Pore Organization in Mesostructured Semiconductors Based on the $\left[\mathrm{SnSe}_{4}\right]^{4-}$ Anion. Nature 2001, 410, 671-675.

(5) Bag, S.; Trikalitis, P. N.; Chupas, P. J.; Armatas, G. S.; Kanatzidis, M. G. Porous Semiconducting Gels and Aerogels from Chalcogenide Clusters. Science 2007, 317, 490-493.

(6) Feng, P.; Bu, X.; Zheng, N. The Interface Chemistry between Chalcogenide Clusters and Open Framework Chalcogenides. Acc. Chem. Res. 2005, 38, 293-303.

(7) Lewis, D. W.; Willock, D. J.; Catlow, C. R. A.; Thomas, J. M.; Hutchings, G. J. De Novo Design of Structure Directing Agents for the Synthesis of Microporous Solids. Nature 1996, 382, 604-606.

(8) Jiang, J.; Jorda, J. L.; Diaz-Cabanas, M. J.; Yu, J.; Corma, A. The Synthesis of an Extra-Large-Pore Zeolite with Double Three-Ring Building Units and a Low Framework Density. Angew. Chem., Int. Ed. 2010, 49, 4986-4988.

(9) Corma, A.; Díaz-Cabañas, M. J.; Martínez-Triguero, J.; Rey, F.; Rius, J. A Large-Cavity Zeolite with Wide Pore Windows and Potential as an Oil Refining Catalyst. Nature 2002, 418, 514-517.

(10) Armatas, G. S.; Kanatzidis, M. G. Mesoporous Germanium-Rich Chalcogenido Frameworks with Highly Polarizable Surfaces and Relevance to Gas Separation. Nat. Mater. 2009, 8, 217-222.

(11) Bag, S.; Kanatzidis, M. G. Chalcogels: Porous MetalChalcogenide Networks from Main-Group Metal Ions. Effect of Surface Polarizability on Selectivity in Gas Separation. J. Am. Chem. Soc. 2010, 132, 14951-14959.

(12) Santner, S.; Dehnen, S. $\left[\mathrm{M}_{4} \mathrm{Sn}_{4} \mathrm{Se}_{17}\right]^{10-}$ Cluster Anions ( $\mathrm{M}=$ $\mathrm{Mn}, \mathrm{Zn}, \mathrm{Cd}$ ) in a $\mathrm{Cs}^{+}$Environment and as Ternary Precursors for Ionothermal Treatment. Inorg. Chem. 2015, 54, 1188-1190.

(13) Lin, Y.; Dehnen, S. [BMIm $]_{4}\left[\mathrm{Sn}_{9} \mathrm{Se}_{20}\right]$ : Ionothermal Synthesis of a Selenidostannate with a 3D Open-Framework Structure. Inorg. Chem. 2011, 50, 7913-7915.
(14) Jiao, F.; Jumas, J. C.; Womes, M.; Chadwick, A. V.; Harrison, A.; Bruce, P. G. Synthesis of Ordered Mesoporous $\mathrm{Fe}_{3} \mathrm{O}_{4}$ and $\gamma-\mathrm{Fe}_{2} \mathrm{O}_{3}$ with Crystalline Walls Using Post-Template Reduction/Oxidation. J. Am. Chem. Soc. 2006, 128, 12905-12909.

(15) Hu, D.-D.; Lin, J.; Zhang, Q.; Lu, J.-N.; Wang, X.-Y.; Wang, Y.W.; Bu, F.; Ding, L.-F.; Wang, L.; Wu, T. Multi-Step Host-Guest Energy Transfer Between Inorganic Chalcogenide-Based Semiconductor Zeolite Material and Organic Dye Molecules. Chem. Mater. 2015, 27, 4099-4104

(16) Lin, J.; Dong, Y.; Zhang, Q.; Hu, D.; Li, N.; Wang, L.; Liu, Y.; $\mathrm{Wu}, \mathrm{T}$. Interrupted Chalcogenide-Based Zeolite-Analog Semiconductor: Atomically Precise Doping for Tunable Electro-/Photoelectrochemical Properties. Angew. Chem. 2015, 127, 5192-5196.

(17) Arachchige, I. U.; Kanatzidis, M. G. Anomalous Band Gap Evolution from Band Inversion in $\mathrm{Pb}_{1-\mathrm{x}} \mathrm{Sn}_{\mathrm{x}}$ Te Nanocrystals. Nano Lett. 2009, 9, 1583-1587.

(18) Lin, Q.; Bu, X.; Mao, C.; Zhao, X.; Sasan, K.; Feng, P. Mimicking High-Silica Zeolites: Highly Stable Germanium- and TinRich Zeolite-Type Chalcogenides. J. Am. Chem. Soc. 2015, 137, 61846187.

(19) Davies, D. W.; Butler, K. T.; Jackson, A. J.; Morris, A.; Frost, J. M.; Skelton, J. M.; Walsh, A. Computational Screening of All Stoichiometric Inorganic Materials. Chem. 2016, 1, 617-627.

(20) Butler, K. T.; Frost, J. M.; Skelton, J. M.; Svane, K. L.; Walsh, A. Computational Materials Design of Crystalline Solids. Chem. Soc. Rev. 2016, 45, 6138-6146.

(21) Zwijnenburg, M. A.; Bromley, S. T.; Foster, M. D.; Bell, R. G.; Delgado-Friedrichs, O.; Jansen, J. C.; Maschmeyer, T. Toward Understanding the Thermodynamic Viability of Zeolites and Related Frameworks through a Simple Topological Model. Chem. Mater. 2004, $16,3809-3820$.

(22) Corma, A.; Rey, F.; Rius, J.; Sabater, M. J.; Valencia, S. Supramolecular Self-Assembled Molecules as Organic Directing Agent for Synthesis of Zeolites. Nature 2004, 431, 287-290.

(23) Couves, J. W.; Jones, R. H.; Parker, S. C.; Tschaufeser, P.; Catlow, C. R. A. Experimental Verification of a Predicted Negative Thermal Expansivity of Crystalline Zeolites. J. Phys.: Condens. Matter 1993, 5, L329-L332.

(24) Foster, M. D.; Rivin, I.; Treacy, M. M. J.; Friedrichs, O. D. A Geometric Solution to the Largest-Free-Sphere Problem in Zeolite Frameworks. Microporous Mesoporous Mater. 2006, 90, 32-38.

(25) O'Keeffe, M.; Yaghi, O. M. Germanate Zeolites: Contrasting the Behavior of Germanate and Silicate Structures Built from Cubic $\mathrm{T}_{8} \mathrm{O}_{20}$ Units ( $\mathrm{T}=\mathrm{Ge}$ or Si). Chem. - Eur. J. 1999, 5, 2796-2801.

(26) Piccione, P. M.; Laberty, C.; Yang, S.; Camblor, M. A.; Navrotsky, A.; Davis, M. E. Thermochemistry of Pure-Silica Zeolites. J. Phys. Chem. B 2000, 104, 10001-10011.

(27) Goldschmidt, V. M. The Principles of Distribution of Chemical Elements in Minerals and Rocks. J. Chem. Soc. 1937, 0, 655-673.

(28) Sastre, G.; Corma, A. Predicting Structural Feasibility of Silica and Germania Zeolites. J. Phys. Chem. C 2010, 114, 1667-1673.

(29) Gillespie, R. J. The Valence-Shell Electron-Pair Repulsion (VSEPR) Theory of Directed Valency. J. Chem. Educ. 1963, 40, 295.

(30) Li, H. Supertetrahedral Sulfide Crystals with Giant Cavities and Channels. Science 1999, 283, 1145-1147.

(31) Hughes, J. T.; Navrotsky, A. MOF-5: Enthalpy of Formation and Energy Landscape of Porous Materials. J. Am. Chem. Soc. 2011, 133, 9184-9187.

(32) Feng, P.; Bu, X.; Zheng, N. The Interface Chemistry between Chalcogenide Clusters and Open Framework Chalcogenides. Acc. Chem. Res. 2005, 38, 293-303.

(33) Mohanan, J. L.; Arachchige, I. U.; Brock, S. L. Porous Semiconductor Chalcogenide Aerogels. Science 2005, 307, 397-400.

(34) Li, H.; Eddaoudi, M.; Laine, A.; O’Keeffe, M.; Yaghi, O. M. Noninterpenetrating Indium Sulfide Supertetrahedral Cristobalite Framework. J. Am. Chem. Soc. 1999, 121, 6096-6097.

(35) Luc, W.; Jiao, F. Synthesis of Nanoporous Metals, Oxides, Carbides, and Sulfides: Beyond Nanocasting. Acc. Chem. Res. 2016, 49, $1351-1358$. 
(36) Zwijnenburg, M. A.; Corà, F.; Bell, R. G. Dramatic Differences between the Energy Landscapes of $\mathrm{SiO}_{2}$ and $\mathrm{SiS}_{2}$ Zeotype Materials. J. Am. Chem. Soc. 2007, 129, 12588-12589.

(37) Butler, K. T.; Hendon, C. H.; Walsh, A. Electronic Structure Modulation of Metal-Organic Frameworks for Hybrid Devices. ACS Appl. Mater. Interfaces 2014, 6, 22044-22050.

(38) Sun, L.; Hendon, C. H.; Minier, M. A.; Walsh, A.; Dinca, M. Million-Fold Electrical Conductivity Enhancement in $\mathrm{Fe}_{2}$ (DEBDC) versus $\mathrm{Mn}_{2}(\mathrm{DEBDC})(\mathrm{E}=\mathrm{S}, \mathrm{O})$. J. Am. Chem. Soc. 2015, 137, 61646167.

(39) Hendon, C. H.; Walsh, A. Chemical Principles Underpinning the Performance of the Metal-organic Framework HKUST-1. Chem. Sci. 2015, 6, 3674-3683.

(40) McDonald, T. M.; Mason, J. A.; Kong, X.; Bloch, E. D.; Gygi, D.; Dani, A.; Crocellà, V.; Giordanino, F.; Odoh, S. O.; Drisdell, W. S.; Vlaisavljevich, B.; Dzubak, A. L.; Poloni, R.; Schnell, S. K.; Planas, N.; Lee, K.; Pascal, T.; Wan, L. F.; Prendergast, D.; Neaton, J. B.; Smit, B.; Kortright, J. B.; Gagliardi, L.; Bordiga, S.; Reimer, J. A.; Long, J. R. Cooperative Insertion of $\mathrm{CO}_{2}$ in Diamine-Appended Metal-Organic Frameworks. Nature 2015, 519, 303-308.

(41) Park, S. S.; Hontz, E. R.; Sun, L.; Hendon, C. H.; Walsh, A.; Van Voorhis, T.; Dinca, M. Cation-Dependent Intrinsic Electrical Conductivity in Isostructural Tetrathiafulvalene-Based Microporous Metal-Organic Frameworks. J. Am. Chem. Soc. 2015, 137, 1774-1777.

(42) Wahila, M. J.; Butler, K. T.; Lebens-Higgins, Z. W.; Hendon, C. H.; Nandur, A. S.; Treharne, R. E.; Quackenbush, N. F.; Sallis, S.; Mason, K.; Paik, H.; Schlom, D. G.; Woicik, J. C.; Guo, J.; Arena, D. A.; White, B. E.; Watson, G. W.; Walsh, A.; Piper, L. F. J. Lone-Pair Stabilization in Transparent Amorphous Tin Oxides: A Potential Route to P-Type Conduction Pathways. Chem. Mater. 2016, 28, $4706-4713$.

(43) Hinuma, Y.; Hatakeyama, T.; Kumagai, Y.; Burton, L. A.; Sato, H.; Muraba, Y.; Iimura, S.; Hiramatsu, H.; Tanaka, I.; Hosono, H.; Oba, F. Discovery of Earth-Abundant Nitride Semiconductors by Computational Screening and High-Pressure Synthesis. Nat. Commun. 2016, 7, 11962.

(44) Butler, K. T.; Hendon, C. H.; Walsh, A. Electronic Chemical Potentials of Porous Metal-Organic Frameworks. J. Am. Chem. Soc. 2014, 136, 2703-2706.

(45) Born, M.; Huang, K. Dynamical Theory of Crystal Lattices; Clarendon Press, 1998.

(46) Sallis, S.; Butler, K. T.; Quackenbush, N. F.; Williams, D. S.; Junda, M.; Fischer, D. a.; Woicik, J. C.; Podraza, N. J.; White, B. E.; Walsh, A.; Piper, L. F. J. Origin of Deep Subgap States in Amorphous Indium Gallium Zinc Oxide: Chemically Disordered Coordination of Oxygen. Appl. Phys. Lett. 2014, 104, 232108.

(47) Zwijnenburg, M. A.; Bromley, S. T. Structure Direction in Zinc Oxide and Related Materials by Cation Substitution: An Analogy with Zeolites. J. Mater. Chem. 2011, 21, 15255-15261.

(48) Vegard, L. Die Konstitution Der Mischkristalle Und Die Raumfüllung Der Atome. Eur. Phys. J. A 1921, 5, 17-26.

(49) Hamilton, B. B.; Rimmer, J. S.; Anderson, M.; Leigh, D. White Light Emission from $\mathrm{C}_{60}$ Molecules Confined in Molecular Cage Materials. Adv. Mater. 1993, 5, 583-585.

(50) Fenwick, O.; Coutiño-Gonzalez, E.; Grandjean, D.; Baekelant, W.; Richard, F.; Bonacchi, S.; De Vos, D.; Lievens, P.; Roeffaers, M.; Hofkens, J.; Samorì, P. Tuning the Energetics and Tailoring the Optical Properties of Silver Clusters Confined in Zeolites. Nat. Mater. 2016, 15, 1017-1022.

(51) Hautier, G.; Miglio, A.; Ceder, G.; Rignanese, G.-M.; Gonze, X. Identification and Design Principles of Low Hole Effective Mass PType Transparent Conducting Oxides. Nat. Commun. 2013, 4, 2292.

(52) Butler, K. T. Morphological Control of Band Offsets for Transparent Bipolar Heterojunctions: The Bädeker Diode. Phys. Status Solidi A 2015, 212, 1461-1465.

(53) Wahila, M. J.; Lebens-Higgins, Z. W.; Quackenbush, N. F.; Nishitani, J.; Walukiewicz, W.; Glans, P.-A.; Guo, J.-H.; Woicik, J. C.; Yu, K. M.; Piper, L. F. J. Evidence of Extreme Type-III Band Offset at
Buried N -Type CdO/P-Type SnTe Interfaces. Phys. Rev. B: Condens. Matter Mater. Phys. 2015, 91, 205307.

(54) Pitanti, A.; Ercolani, D.; Sorba, L.; Roddaro, S.; Beltram, F.; Nasi, L.; Salviati, G.; Tredicucci, A. InAs/InP/InSb Nanowires as Low Capacitance N - N Heterojunction Diodes. Phys. Rev. X 2011, 1, 11006.

(55) Chen, C. Y.; Shik, A.; Pitanti, A.; Tredicucci, A.; Ercolani, D.; Sorba, L.; Beltram, F.; Ruda, H. E. Electron Beam Induced Current in InSb-InAs Nanowire Type-III Heterostructures. Appl. Phys. Lett. 2012, 101, 63116.

(56) Zhang, X.; Zhang, L.; Perkins, J. D.; Zunger, A. Intrinsic Transparent Conductors without Doping. Phys. Rev. Lett. 2015, 115, 176602.

(57) Lim, H. E.; Miyata, Y.; Fujihara, M.; Okada, S.; Liu, Z.; Arifin; Sato, K.; Omachi, H.; Kitaura, R.; Irle, S.; Suenaga, K.; Shinohara, H. Fabrication and Optical Probing of Highly Extended, Ultrathin Graphene Nanoribbons in Carbon Nanotubes. ACS Nano 2015, 9, 5034-5040.

(58) Talin, A.; Centrone, A.; Ford, A. C.; Foster, M. E.; Stavila, V.; Haney, P.; Kinney, R. A.; Szalai, V.; El Gabaly, F.; Yoon, H. P.; Léonard, F.; Allendorf, M. D. Tunable Electrical Conductivity in Metal-Organic Framework Thin-Film Devices. Science 2014, 343, $66-$ 69.

(59) Benz, S.; López-andarias, J.; Mareda, J.; Sakai, N.; Matile, S. Catalysis with Chalcogen Bonds Communications. Angew. Chem., Int. Ed. 2017, 56, 812-815.

(60) Regulacio, M. D.; Han, M. Multinary I-III-VI ${ }_{2}$ and $\mathrm{I}_{2}-\mathrm{II}-\mathrm{IV}^{-\mathrm{VI}_{4}}$ Semiconductor Nanostructures for Photocatalytic Applications. Acc. Chem. Res. 2016, 49, 511-519.

(61) Gounder, R.; Iglesia, E. The Roles of Entropy and Enthalpy in Stabilizing Catalysis. Acc. Chem. Res. 2012, 45, 229-238.

(62) Butler, K. T.; Walsh, A.; Cheetham, A. K.; Kieslich, G. Organised Chaos: Entropy in Hybrid Inorganic-Organic Systems and Other Materials. Chem. Sci. 2016, 7, 6316-6324.

(63) Na, K.; Choi, K. M.; Yaghi, O. M.; Somorjai, G. A. Metal Nanocrystals Embedded in Single Nanocrystals of MOFs Give Unusual Selectivity as Heterogeneous Catalysts. Nano Lett. 2014, 14, 5979-5983.

(64) Milla, S.; Depiereux, S.; Kestemont, P. The Effects of Estrogenic and Androgenic Endocrine Disruptors on the Immune System of Fish: A Review. Ecotoxicology 2011, 20, 305-319.

(65) Colborn, T.; Saal, F. S.; Soto, A. M. Developmental Effects of Endocrine-Disrupting Chemicals in Wildlife and Humans. Enviromental Heal. Perspect. 1993, 101, 378-384.

(66) Viswanathan, B.; Subramanian, V.; Lee, J. S. Materials and Processes for Solar Fuel Production; Lockwood, D. J., Ed.; Springer, 2014.

(67) Linic, S.; Aslam, U.; Boerigter, C.; Morabito, M. Photochemical Transformations on Plasmonic Metal Nanoparticles. Nat. Mater. 2015, $14,567-576$.

(68) Boerigter, C.; Aslam, U.; Linic, S. Mechanism of Charge Transfer from Plasmonic Nanostructures to Chemically Attached. ACS Nano 2016, 10, 6108-6115. 